\title{
Численное моделирование субнаносекундного лавинного переключения кремниевых $n^{+}-n-n^{+}$-структур
}

\author{
(С) Н.И. Подольская, П.Б. Родин \\ Физико-технический институт им.А.Ф. Иоффе Российской академии наук, \\ 194021 Санкт-Петербург, Россия \\ E-mail: natalia@scc.ioffe.ru
}

(Получена 17 октября 2018 г. Принята к печати 25 октября 2018 г.)

\begin{abstract}
Приведены результаты численного моделирования недавно открытого явления - субнаносекундного лавинного переключения кремниевых $n^{+}-n-n^{+}$-структур, инициированного быстро нарастающим высоковольтным импульсом. Показано, что электрическое поле в $n^{+}-n-n^{+}$-структуре на этапе роста напряжения практически однородно вдоль направления тока и достигает эффективного порога ударной ионизации $\sim 200$ кВ/см на всей толщине структуры. Сравнение результатов численного моделирования и экспериментов указывает на однородность распределения поля и в поперечном к току направлении. Таким образом, сверхбыстрый лавинный пробой происходит квазиоднородно во всем объеме структуры. Время переключения составляет $\sim 150$ пс. Проведено сравнение результатов расчетов для разных моделей ударной ионизации. Даны оценки параметров инициирующего импульса напряжения, необходимого для переключения кремниевых $n^{+}-n-n^{+}$-структур.
\end{abstract}

DOI: $10.21883 /$ FTP.2019.03.47294.8984

\section{1. Введение}

Известно, что быстронарастающий высоковольтный импульс, приложенный к диодной $p^{+}-n-n^{+}$-структуре, приводит к лавинному переключению в проводящее состояние за время около 100 пс [1]. Это явление обнаружено и исследовано в кремниевых и арсендигаллиевых диодах [1-4] и получило название задержанного лавинного пробоя обратно смещенных $p-n$-переходов [1]. Такое переключение называют также сверхбыстрым, поскольку время переключения на порядок меньше времени, за которое двигающийся с насыщенной дрейфовой скоростью носитель может пролететь через базу структуры. Время нарастания инициирующего задержанный пробой импульса составляет единицы наносекунды, а время переключение диода - доли наносекунды, т.е. диод способен обострять мощные высоковольтные импульсы. Основанные на задержанном ударноионизационном пробое приборы - кремниевые обострительные диоды и динисторы - с успехом применяется в импульсной полупроводниковой электронике большой мощности [5-7].

Недавно было экспериментально установлено [8-10], что сверхбыстрое лавинное переключение, инициированное быстронарастающим высоковольтным импульсом, также возможно и в полупроводниковых $n^{+}-n-n^{+}$-структурах. В таких структурах отсутствует переход, и соответственно они не обладают способностью блокировать стационарное напряжение. Тем не менее при скорости нарастания инициирующего импульса более $10 \mathrm{\kappa B} / \mathrm{Hc}$ напряжение на кремниевой $n^{+}-n-n^{+}$-структуре общей толщиной 100-200 мкм перед переключением достигает 2-3 кВ [8-10]. Такое напряжение отвечает средней напряженности электрического поля, близкой к эффективному порогу ударной ионизации в кремнии и составляющей 200 кВ/см. Измеренное характерное время переключения структуры составляет 150 пс [8-10]. Помимо кремниевых структур, успешное переключение в проводящее состояние экспериментально наблюдалось также для структур на основе $\mathrm{ZnSe}$, которые представляли собой объемные образцы с омическими контактами большой площади [8]. Эксперименты [8-10] показывают, что инициированное быстронарастающим импульсом лавинное переключение представляет собой универсальный метод создания относительно больших (порядка кубического миллиметра) объемов неравновесной электронно-дырочной плазмы в полупроводниках. Этот метод применим в широкозонных прямозонных полупроводниках и потому представляет потенциальный интерес для оптических приложений.

Экспериментальное наблюдение физических процессов, протекающих при переключении кремниевых структур в режиме задержанного ударно-ионизационного пробоя, чрезвычайно затруднено. Это обусловлено малой $(\sim 100$ пс) длительностью переходного процесса и низкой эффективностью оптической рекомбинации в непрямозонном кремнии, не позволяющей определить динамику распространения неравновесной электроннодырочной плазмы в приборе на данном временно́м масштабе. Как следствие, численное моделирование оказывается особенно важным для понимания физики переключения этого класса быстродействующих импульсных приборов. В настоящей работе проведено моделирование сверхбыстрого лавинного переключения кремниевых $n^{+}-n-n^{+}$-структур с помощью программного обеспечения TCAD Silvaco [11]. Показано, что численное моделирование в диффузионно-дрейфовом приближении позволяет получить количественное согласие с экспериментом и описать физическую картину сверхбыст- 
рого переключения. Проведено сравнение результатов для различных аппроксимаций коэффициентов ударной ионизации. Определены необходимые для сверхбыстрого переключения скорость нарастания и амплитуда инициирующего импульса напряжения.

\section{2. Модель}

Времени переключения 100 пс отвечает электромагнитная длина $\sim 3 \mathrm{~cm}$, меньшая характерного размера измерительной схемы. В силу этого регистрация переходных процессов требует применения согласованных высокочастотных измерительных трактов. В экспериментах [8-10] применялся специально сконструированный резистивный ответвитель (см. [12]), обеспечивающий возможность одновременной и независимой регистрации напряжения на исследуемой структуре и тока через нее с высоким временни́м разрешением (не хуже 50 пс). Простейшая реальная схема, обеспечивающая измерение только тока, протекающего через структуру, предполагает включение исследуемой структуры в разрыв центральной жилы $50 \Omega$ согласованного коаксиального тракта. При моделировании использовалась электрическая схема с сосредоточенными элементами, показанная на вставке к рис. 1 . Здесь $U_{\text {in }}(t)-$ запускающий импульс внешнего генератора, $U_{s}(t)-$ напряжение на структуре, $I(t)-$ ток через структуру и нагрузку $R$. Для модельной электрической схемы, показанной на вставке к рис. 1, в отличие от реальной схемы с коаксиальными трактами выполняется уравнение Кирхгофа $U_{\text {in }}(t)=U_{s}(t)+R \cdot I(t)$. Поэтому при сравнении результатов наших расчетов с экспериментальными данными в качестве $U_{\text {in }}(t)$ было выбрано измеренное в эксперименте напряжение на структуре вместе с нагрузкой. При вычислении критических значений скорости роста и амплитуды запускающего импульса для $U_{\text {in }}(t)$ принята кусочно-линейная зависимость, составленная из линейно нарастающей части, переходящей в горизонтальную полку.

Выбранные при моделировании профили легирования (см. вставку на рис. 2) отвечают структурам, экспериментально исследованным в работах [8-10]. Общая толщина структуры составляет $W \sim 200$ мкм, площадь $S \sim 1.0$ мм $^{2}$, концентрация легирующей примеси в $n$-слое $N_{d}=1.7 \cdot 10^{14} \mathrm{~cm}^{-3}$. Следуя экспериментальным работам [8-10], мы обозначаем структуры с узким ( $\sim 100$ мкм $) \quad n$-слоем как тип А, а структуры с широким ( 180 мкм) $n$-слоем - как тип В. Точность определения толщин слоев исследуемых структур не лучше 10 мкм [8]. Поэтому при численном моделировании толщины слоев рассматривались как подгоночные параметры, варьируемые в этих пределах для достижения наилучшего согласия с экспериментальным данными.

Динамика носителей и электрического поля в структуре была получена решением диффузионно-дрейфовых

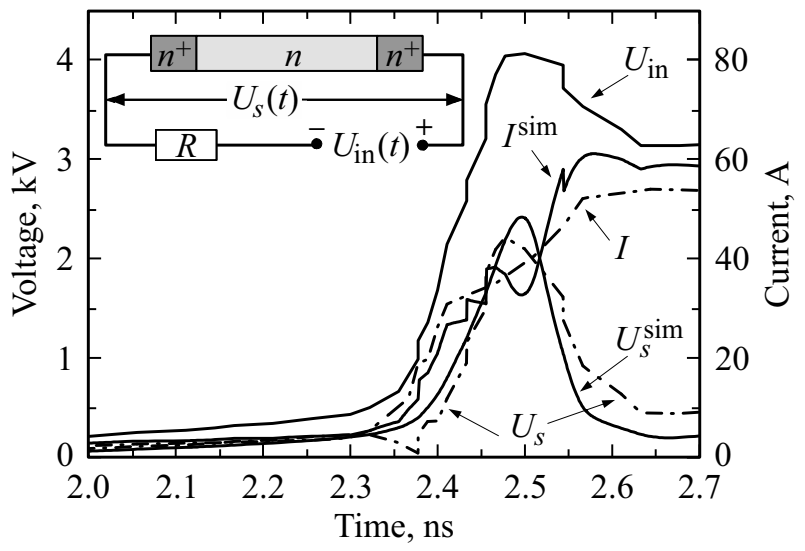

Рис. 1. Субнаносекундное переключение кремниевой $n^{+}-n-n^{+}$-структуры типа А с толщинами $n^{+}$-слоев 55 мкм и $n$-слоя 90 мкм. Зависимости от времени напряжения на структуре $U_{s}^{\mathrm{sim}}(t)$ и тока $I^{\mathrm{sim}}(t)$, полученные численным моделированием, приведены вместе с измеренными экспериментально [8] зависимостями $U_{s}(t)$ и $I(t)$, а также экспериментально измеренной зависимостью напряжения на структуре вместе с нагрузкой $U_{\text {in }}(t)$. На вставке - модельная электрическая цепь.

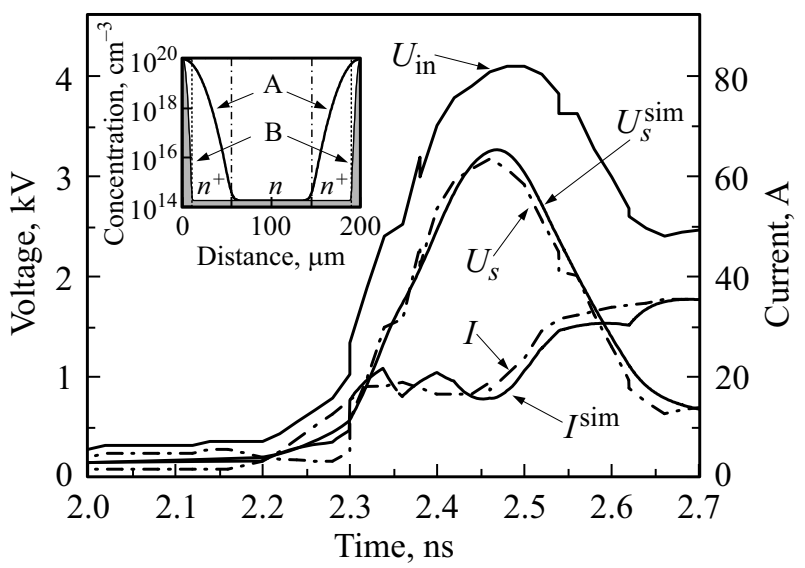

Рис. 2. Субнаносекундное переключение кремниевой $n^{+}-n-n^{+}$-структуры типа В с толщинами $n^{+}$-слоев 10 мкм и слоя 160 мкм. Зависимости от времени напряжения на структуре $U_{s}^{\text {sim }}(t)$ и тока $I^{\text {sim }}(t)$, полученные численным моделированием, приведены вместе с измеренными экспериментально [8] зависимостями $U_{s}(t)$ и $I(t)$, а также экспериментально измеренной зависимостью напряжения на структуре вместе с нагрузкой $U_{\text {in }}(t)$. На вставке - профили легирования структур типа А и В.

транспортных уравнений совместно с уравнением Пуассона и уравнением Кирхгофа для показанной на вставке к рис. 1 электрической цепи. Температура считалась комнатной, моделирование проводилось в одномерном приближении. Чтобы установить применимость различных аппроксимаций коэффициентов ударной ионизации, при описании быстрых ударно-ионизационных процессов в импульсных лавинных приборах расчеты были 

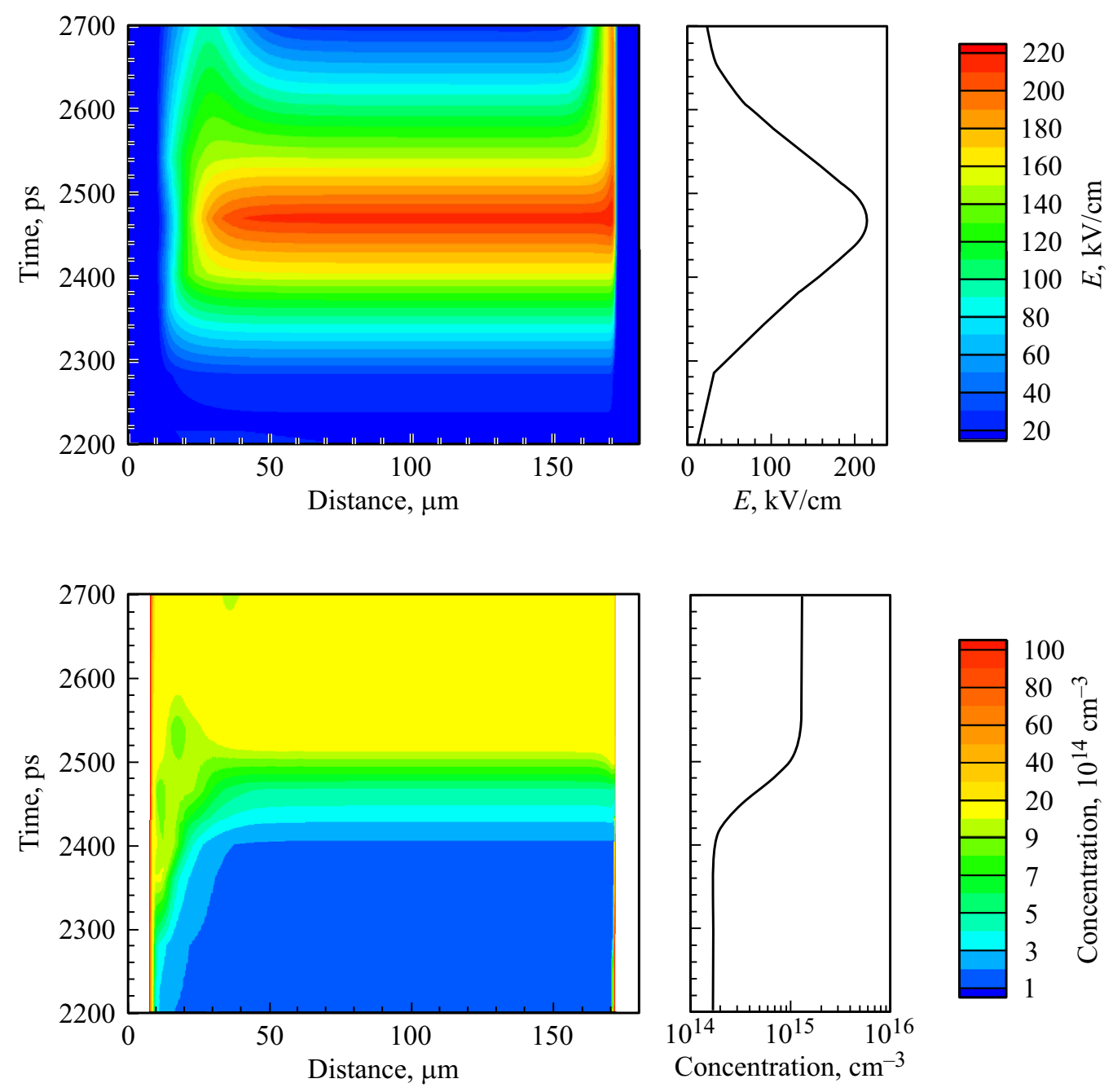

Рис. 3. Пространственно-временна́я динамика электрического поля и концентрации электронов в $n$-слое кремниевой $n^{+}-n-n^{+}$-структуры типа В с толщинами $n^{+}$-слоя 10 мкм и $n$-слоя 160 мкм при субнаносекундном лавинном переключении (см. рис. 2). Катод расположен слева. Кривые справа показывают временну́ю динамику поля и концентрации в середине $n$-слоя. Цветная версия рисунка - в электронной версии статьи.

проведены как для имплементированных в программный пакет TCAD Silvaco [11], так и для других известных из литературы моделей ударной ионизации.

\section{3. Результаты моделирования}

На рис. 1 и 2 показаны зависимости напряжения на структуре и тока в цепи от времени для $n^{+}-n-n^{+}$-структур типа А и типа В (соответствующие профили легирования показаны на вставке к рис. 2), полученные численным моделированием. Применялась аппроксимация [13-15] для коэффициентов ударной ионизации, имплементированная в TCAD Silvaco [11]. Представленные кривые соответствуют толщинам $n^{+}$-слоев 55 мкм и толщине $n$-слоя 90 мкм для структуры типа А. Для структуры типа В эти толщины составляли 10 и 160 мкм соответственно. Отметим, что такой выбор толщин слоев позволяет хорошо описать динамику напряжения на структуре при всех экспериментально исследованных режимах переключения [8].

Время переключения структур обоих типов составляет около $\sim 150$ пс, что близко ко времени нарастания приложенного импульса напряжения. Время нарастания тока $(\sim 300$ пс $)$ превосходит время нарастания приложенного импульса. Таким образом, лавинное переключение $n^{+}-n-n^{+}$-структур не приводит к обострению приложенного импульса, что принципиально отличает их от диодных структур с $p-n$-переходом. Вместе с тем остаточное напряжение $\sim 500 \mathrm{~B}$ и соответственно амплитуда тока практически такие же, как у кремниевых диодных структур $[3,4,12]$. Это указывает на сравнимые концентрации неравновесных носителей, созданных ударной ионизацией. Хорошее согласие одномерного численного моделирования с экспериментом указывает на однородность процесса лавинного переключения по площади прибора. 
Пространственно-временна́я динамика электрического поля и концентрация электронов в $n$-области показана на рис. 3 на примере $n^{+}-n-n^{+}$-структуры типа В с толщинами $n^{+}$-слоев 10 мкм и $n$-слоя 160 мкм. Концентрация дырок после переключения совпадает с концентрацией электронов. Видно, что электрическое поле возрастает квазиоднородно во всей $n$-области. Объемный заряд, обеспечивающий рост электрического поля, создается инжекцией электронов в $n$-область из левого $n^{+}$-слоя (толщина этого слоя отрицательного заряда $\sim 10$ мкм) и экстракцией из правого $n^{+}$-слоя структуры (толщина этого слоя положительного заряда несколько микрометров). Пространственная разделенность этих двух разноименно заряженных узких слоев и обеспечивает однородность электрического поля в структуре. К моменту начала пробоя напряженность достигает 200 кВ/см. Пробой развивается квазиоднородно в направлении тока, заполняя структуру неравновесной электронно-дырочной плазмой с концентрацией $10^{15} \mathrm{~cm}^{-3}$.

\section{4. Влияние аппроксимаций коэффициентов ударной ионизации на результаты численного моделирования}

При динамическом лавинном пробое ударно-ионизационное размножение носителей происходит в условиях субнаносекундного роста напряженности электрического поля от нуля до $200 \mathrm{\kappa B} / \mathrm{cm}$. Таким образом, для количественно верного теоретического описания этого процесса необходима зависимость коэффициентов ударной ионизации, адекватная в широком диапазоне напряженностей электрического поля. В литературе имеются различные аппроксимации [13-23], большая часть из которых имплементирована в программное обеспечение TCAD Silvaco [13-22].

На рис. 4 приведены напряжения на кремниевой $n^{+}-n-n^{+}$структуре с профилем легирования типа В для различных аппроксимаций, полученные численным моделированием. Видно, что моделирование с применением аппроксимаций [13-15] (кривая 2), [16] (кривая 3), [17] (кривая 4), [18,19] (кривая 5), [23] (кривая 6) приводят к близким результатам. Аппроксимации [20] (кривая 7) и [21] (кривая 8) завышают напряжение динамического пробоя структуры. Причиной этого являются меньшие, чем для аппроксимаций [13-19,23], значения коэффициентов ударной ионизации для электронов в электрических полях напряженностью менее 300 кВ/см. Особняком стоят результаты, полученные для аппроксимации [22] в том виде, как она имплементирована в TCAD Silvaco (кривая 9 на рис. 4). Видно, что кривая 9 практически совпадает с кривой 1 , отвечающей результатам моделирования, полученным без учета ударной ионизации. Анализ результатов показал, что это связано не с самой аппроксимацией [22], а с ее

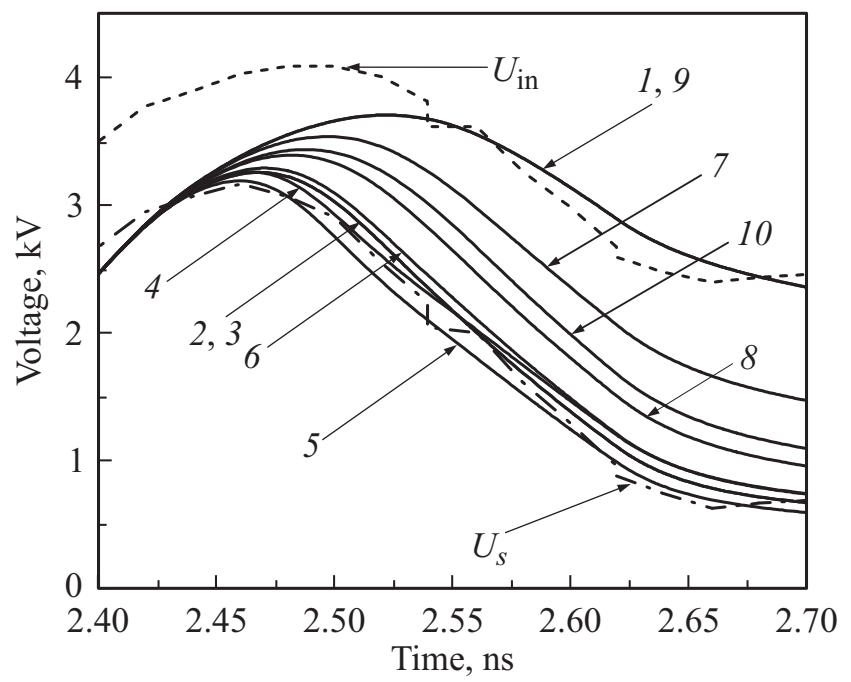

Рис. 4. Зависимости напряжения на структуре типа В с толщинами $n^{+}$- слоя 10 мкм и $n$-слоя 160 мкм, полученные численным моделированием для различных аппроксимаций коэффициентов ударной ионизации. Референтная кривая 1 соответствует результатам моделирования без учета ударной ионизации. Кривые 2-8 отвечают аппроксимациям [13-15] (кривая 2), [16] (кривая 3), [17] (кривая 4), [18,19] (кривая 5), [23] (кривая 6), [20] (кривая 7), [21] (кривая 8) соответственно. Кривая 9 соответствует результатам, полученным с аппроксимацией [22] в той форме, как она была имплементирована разработчиками программного обеспечения в TCAD Silvaco [11]. Кривая 10 была получена путем программной реализации модели [22], выполненной авторами данной работы и подключенной для использования при моделировании в TCAD Silvaco вместо исходной версии реализации этой модели. Кривые $U_{s}$ и $U_{\text {in }}$ демонстрируют эспериментально измеренные [8] зависимости от времени напряжения на структуре и на структуре вместе с нагрузкой соответственно.

имплементацией разработчиками программного обеспечения в TCAD Silvaco [11]. Кривая 10 на рис. 4 получена путем программной реализации модели [22], выполненной авторами данной работы и подключенной в TCAD Silvaco вместо исходной версии реализации этой модели. Сравнение кривых 9 и 10 показывает, что в случае кривой 9 интенсивность ударной ионизации явно недооценена. Вследствие этого численное моделирование предсказывает развитие пробоя при существенно бо́льших значениях электрического поля, чем это наблюдается в эксперименте. Отметим, что тем не менее кривая 10 демонстрирует некоторую недооценку коэффициентов ударной ионизации, вычисленных в рамках модели [22]. Применение аппроксимации [22] в той форме, как она была исходно имплементирована разработчиками программного обеспечения TCAD Silvaco, в работе [24] привело к количественно неверным предсказаниям для напряжения динамического пробоя и величины остаточного напряжения в $n^{+}-n-n^{+}$-структурах. 


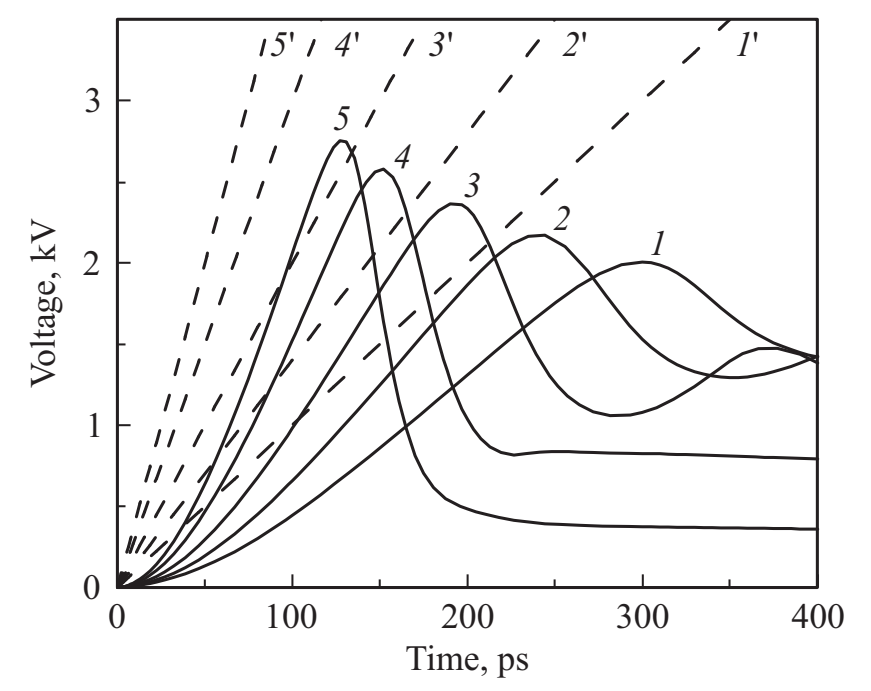

Рис. 5. Напряжение (сплошные линии 1-5) на $n^{+}-n-n^{+}$-структуре, включенной последовательно с $50-\Omega$ нагрузкой с толщинами $n^{+}$-слоев по 7 мкм и $n$-слоя 100 мкм, а также площадью поперечного сечения $1 \mathrm{mм}^{2}$ для линейно нарастающих импульсов приложенного напряжения $U_{\text {in }}$ со скоростями нарастания 10, 14, 20, 30 и 40 кВ/нс (показаны штриховыми линиями $1^{\prime}-5^{\prime}$ соответственно).

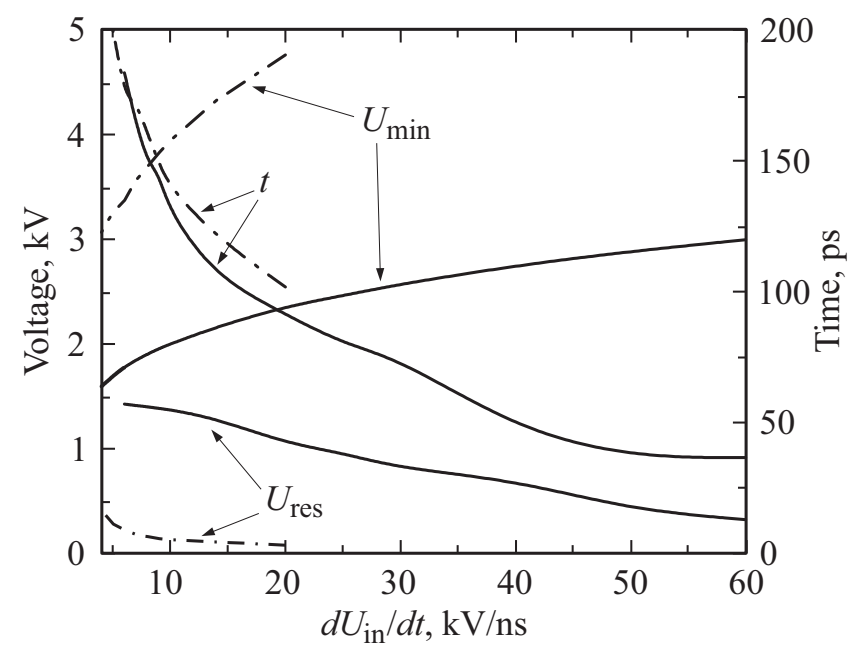

Рис. 6. Зависимости минимально необходимой для сверхбыстрого лавинного переключения амплитуды инициирующего импульса $U_{\min }$, времени переключения структуры в проводящее состояние $t$ и остаточного напряжения $U_{\text {res }}$ от значений скорости нарастания $d U_{\text {in }} / d t$ линейно нарастающих импульсов приложенного напряжения $U_{\text {in }}$ (сплошные линии). Зависимости получены для $n^{+}-n-n^{+}$-структуры с толщинами $n^{+}$-слоев по 7 мкм и $n$-слоя 100 мкм, а также площадью поперечного сечения $1 \mathrm{mм}^{2}$. Сплошные линии соответствуют результатам, полученным численным моделированием с аппроксимацией [13-15]. Штрихпунктирными линиями показаны результаты из работы [24], полученные численным моделированием с аппроксимацией [22] для коэффициентов ударной ионизации в той форме, как она была имплементирована разработчиками программного обеспечения в TCAD Silvaco [11].

\section{5. Результаты моделирования: критические параметры запускающего импульса}

Для сверхбыстрого лавинного переключения $n^{+}-n-n^{+}$-структуры требуются существенно бо́льшая амплитуда и скорость нарастания приложенного импуль$\mathrm{ca}$, чем для диодной $p^{+}-n-n^{+}$-структуры со схожими геометрией и легированием базы. На рис. 5 показаны результаты моделирования для линейно нарастающего импульса $U_{\text {in }}(t)$ и модели ударной ионизации, описанной в работах [13-15]. Численное моделирование проводилось для $n^{+}-n-n^{+}$-структуры с такими же параметрами, что и в работе [24]: толщины $n^{+}$-слоев -7 мкм, $n$-слоя 100 мкм, площадь поперечного сечения $\sim 1$ мм² $^{2}$ Видно, что успешное переключение достигается только при $d U / d t>20 \mathrm{\kappa B} / \mathrm{нc}$, в то время как диодные структуры успешно переключаются уже при $d U / d t>2$ кВ/нс [1-4].

Вследствие задержки развития ударной ионизации напряжение динамического пробоя растет с увеличением скорости нарастания $d U_{\text {in }} / d t$. Это обстоятельство накладывает ограничение на минимальную амплитуду приложенного импульса. На рис. 6 приведены зависимости необходимой для сверхбыстрого лавинного переключения амплитуды импульса, остаточного напряжения и времени переключения от скорости роста приложенного напряжения.

\section{6. Заключение}

Субнаносекундное лавинное переключение кремниевых $n^{+}-n-n^{+}$-структур под действием быстронарастающего импульса высокого напряжения допускает количественно точное описание в рамках одномерной диффузионно-дрейфовой модели, что указывает на высокую степень однородности переключения по площади. Численное моделирование показывает также практически однородное развитие процессов ударной ионизации в направлении тока. Таким образом, можно говорить о квазиоднородном заполнении структуры неравновесной электронно-дырочной плазмой, созданной ударной ионизацией. В этом сверхбыстрый лавинный пробой $n^{+}-n-n^{+}$-структур качественно отличается от исследованных в работе [25] образцов с точечным контактомострием, в которых высоковольтный быстронарастающий импульс напряжения инициирует распространение нитевидных стримеров.

Однородность лавинного пробоя структуры вдоль направления тока обеспечивается квазиоднородным ростом напряженности электрического поля, который в свою очередь обусловлен тем, что в условиях быстрого подъема напряжения слои пространственного заряда создаются только на крае $n$-базы (отрицательный слой, созданный инжекцией в $n$-базу) и в $n^{+}$-области (положительный слой, созданный экстракцией электронов из $n^{+}$-слоя). В соответствии с законом Гаусса 
электрическое поле между этими слоями однородно и быстро достигает пробойного. В совокупности с большой концентрацией равновесных электронов в необедненной n-базе, играющих роль затравочных носителей, это создает все предпосылки для однородного переключения. Данная картина сверхбыстрого переключения $n^{+}-n-n^{+}$-структур была качественно предсказана нами уже в работе [24] до экспериментального обнаружения эффекта [8-10], хотя сделанные в [24] предсказания оказались количественно неверными из-за существенной недооценки коэффициентов ударной ионизации аппроксимации [22] в той форме, как она была исходно имплементирована разработчиками программного обеспечения TCAD Silvaco [11].

Результаты моделирования существенно зависят от выбора модели ударной ионизации. В близком согласии друг с другом и экспериментом оказываются результаты численного моделирования, полученные с аппроксимациями [13-19,23]. Аппроксимации [20-22] недооценивают интенсивность ударной ионизации, что приводит к завышенному значению напряжения динамического пробоя.

Авторы признательны В.И. Брылевскому, И.В. Грехову и И.А. Смирновой за многочисленные полезные обсуждения и помощь. Работы выполнена при поддержке Российского научного фонда, проект № 14-29-00094.

\section{Список литературы}

[1] И.В. Грехов, А.Ф. Кардо-Сысоев. Письма ЖТФ, 5, 950 (1979).

[2] Ж.И. Алферов, И.В. Грехов, В.М. Ефанов, А.Ф. КардоСысоев, В.И. Корольков, М.Н. Степанова. Письма ЖТФ, 13, 1089 (1987).

[3] V.I. Brylevskiy, I.A. Smirnova, A.V. Rozhkov, P.N. Brunkov, P.B. Rodin, I.V. Grekov. IEEE Trans. Plasma Sci., 44, 1941 (2016).

[4] V. Brylevskiy, I. Smirnova, A. Gutkin, P. Brunkov, P. Rodin, I. Grekhov. J. Appl. Phys., 122, 185701 (2017).

[5] R.J. Focia, E. Schamiloghu, C.B. Fleddermann, F.J. Agee, J. Gaudet. IEEE Trans. Plasma Sci., 25, 138 (1997).

[6] A.F. Kardo-Sysoev. Ultra-Wideband Radar Technology, ed. by J.D. Taylor (London-N.Y.-Washington, CRC Press, Boca Raton, 2001).

[7] I.V. Grekhov. IEEE Trans. Plasma Sci., 38, 1118 (2010).

[8] В.И. Брылевский, И.А. Смирнова, Н.И. Подольская, Ю.А. Жарова, П.Б. Родин, И.В. Грехов. Письма ЖТФ, 44, 66 (2018).

[9] V. Brylevskiy, I. Smirnova, N. Podolska, Yu. Zharova, P. Rodin, I. Grekhov. Proc. 2017 IEEE 21st Int. Conf. on Pulsed Power (PPC). (Brighton, UK, 2017) p. 1.

[10] V. Brylevskiy, I. Smirnova, N. Podolska, Yu. Zharova, P. Rodin, I. Grekhov. IEEE Trans. on Plasma Sci., Special Issue on Pulsed Power Science and Technology, 2018, in print.

[11] SILVACO ATLAS, User guide, http://www.silvaco.com

[12] В.И. Брылевский, И.А. Смирнова, П.Б. Родин, И.В. Грехов. Письма ЖТФ, 40, 80 (2014).
[13] S. Selberherr. Analysis and Simulation of Semiconductor Devices (Wien-N.Y., Springer Verlag, 1984).

[14] R. Van Overstraeten, H. De Man. Solid-State Electron., 13, 583 (1970).

[15] P. Mars. Int. J. Electron., 32, 23 (1963).

[16] A.G. Chynoweth. Phys. Rev., 109, 1537 (1958).

[17] C.R. Crowell, S.M. Sze. Appl. Phys. Lett.. 9, 242 (1966).

[18] W.N. Grant. Solid-State Electron., 16, 1189 (1973).

[19] G.A. Baraff. Appl. Phys. Rev., 128, 2507 (1962).

[20] Y. Okuto, R. Crowell. Solid-State Electron., 18, 161 (1975).

[21] T. Lackner. Solid-State Electron., 34, 33 (1991).

[22] M. Valdinoci, D. Ventura, M.C. Vecchi, M. Rudan, G. Baccarani, F. Illien, A. Stricker, L. Zullino. Proc. Int. Conf. Simulations of Semiconductor Processes and Devices (SISPAD 99) (Kyoto, Japan, 1999) p. 27.

[23] S. Reggiani, E. Gnani, M. Rudan, G. Baccarani, Ch. Corvasce, D. Barlini, M. Ciappa, W. Fichtner, M. Denison, N. Jensen, G. Groos, M. Stecher. IEEE Trans. Electron Dev., 52, 2290 (2005).

[24] Н.И. Подольская, П.Б. Родин. Письма ЖТФ, 43, 54 (2017).

[25] Г.А. Месяц, А.С. Насибов, В.Г. Шпак, С.А. Шунайлов, М.И. Яландин. ЖЭТФ, 133, 1162 (2008).

Редактор Г.А. Оганесян

\section{Subnanosecond avalanche switching simulations of $n^{+}-n-n^{+}$-silicon structures}

\section{N.I. Podolska, P.B. Rodin}

loffe Institute, 194021 St. Petersburg, Russia

Abstract Numerical simulations of recently discovered effect of subnanosecond avalanche switching of $\mathrm{Si} n^{+}-n-n^{+}$-structures have been performed. The electric field in $n^{+}-n-n^{+}$-structure is shown to remain quasi-uniform along the current flow direction during the voltage raise stage and it reaches the effective threshold of impact ionization of $\sim 200 \mathrm{kV} / \mathrm{cm}$ in the whole $n$-base. Comparing numerical results with experiments we argue that the field distribution is as well uniform in the transverse direction. Hense, the superfast avalanche transient develops quasi-uniformly in the whole $n$-base volume. The switching time is about $\sim 150 \mathrm{ps}$. We compare numerical results obtained for various impact ionization models and estimate parameters of the initial voltage pulse that are required for superfast avalanche switching of $n^{+}-n-n^{+}$-structures. 